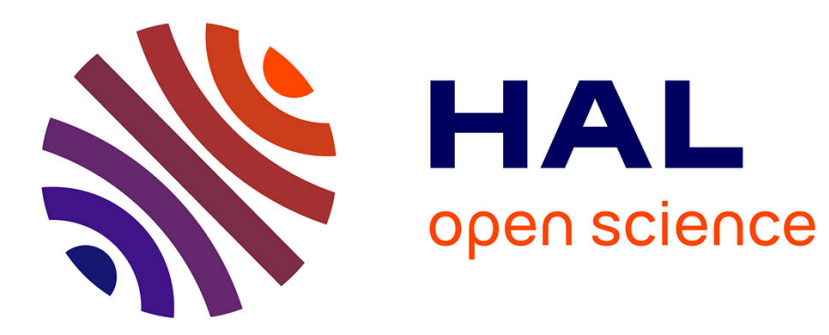

\title{
Distributed Adaptive Sensor Fault Tolerant Control for Smart Buildings
}

Panayiotis Papadopoulos, Vasso Reppa, Marios M. Polycarpou, Christos G. Panayiotou

\section{- To cite this version:}

Panayiotis Papadopoulos, Vasso Reppa, Marios M. Polycarpou, Christos G. Panayiotou. Distributed Adaptive Sensor Fault Tolerant Control for Smart Buildings. 54th IEEE Conference on Decision and Control (CDC), Dec 2015, OSAKA, Japan. 10.1109/cdc.2015.7402690 . hal-01260229

\section{HAL Id: hal-01260229}

https://hal-centralesupelec.archives-ouvertes.fr/hal-01260229

Submitted on 1 Mar 2018

HAL is a multi-disciplinary open access archive for the deposit and dissemination of scientific research documents, whether they are published or not. The documents may come from teaching and research institutions in France or abroad, or from public or private research centers.
L'archive ouverte pluridisciplinaire HAL, est destinée au dépôt et à la diffusion de documents scientifiques de niveau recherche, publiés ou non, émanant des établissements d'enseignement et de recherche français ou étrangers, des laboratoires publics ou privés. 


\title{
Distributed Adaptive Sensor Fault Tolerant Control for Smart Buildings
}

\author{
Panayiotis M. Papadopoulos, Vasso Reppa, Marios M. Polycarpou and Christos G. Panayiotou
}

\begin{abstract}
This paper presents a model-based distributed fault-tolerant control (FTC) scheme with emphasis on compensating the effects of sensor faults in multi-zone heating, ventilating and air-conditioning (HVAC) systems. A bank of local adaptive FTC agents are designed to accommodate possible sensor faults in HVAC systems, modeled as a set of interconnected, nonlinear subsystems. In order to compensate the fault effects that may propagate in the neighboring subsystems, each local FTC utilizes the information generated by a local monitoring agent that diagnoses the sensor faults and performs an adaptive estimation of the isolated sensor faults. The local monitoring and control agents are allowed to exchange information with neighboring agents. Simulation results are used to illustrate the effectiveness of the proposed methodology applied to a sevenzone HVAC system.
\end{abstract}

\section{INTRODUCTION}

In recent years, there has been significant technological activity in the development of smart buildings, which have emerged based on the need to monitor and control the indoor living conditions and safety of the occupants, as well as the energy consumption of large-scale buildings. One of the most essential elements of a smart building, is the heating, ventilating and air-conditioning (HVAC) system. A HVAC can be regarded as a large-scale complex network of interconnected subsystems that consist of several electrical and mechanical components, as well as a large number of sensors. During its operational life, the HVAC components may be impacted by faults, which constitute a key reason for increases in energy consumption due to performance degradation of the system, while they may also lead to uncomfortable conditions for the occupants.

Taking into account the interconnected characteristics of HVAC systems, the early diagnosis and accommodation of faults is critical, since local fault effects may propagate from a local subsystem to neighboring subsystems either through the physical interconnections or through the distributed control scheme. To tackle this problem, the implementation of a fault tolerant control (FTC) scheme [1] is required. Several researchers have developed nominal feedback control [2],

This work was supported by the European Research Council (ERC) under the ERC Advanced Grant FAULT-ADAPTIVE and by the People Programme (Marie Curie Actions) under the REA grant agreement $n^{\circ}$ 626891 (FUTuRISM).

Panayiotis M. Papadopoulos, Marios M. Polycarpou and Christos G. Panayiotou are with KIOS Research Center for Intelligent Systems and Networks, Department of Electrical and Computer Engineering, University of Cyprus, Nicosia, 1678, Cyprus papadopoulos.panagiotis@ucy.ac.cy, mpolycarducy.ac.cy,christospeucy.ac.cy.

Vasso Reppa is with the Laboratory of Signals and Systems (UMR CNRS 8506), CentraleSupélec-CNRS-University Paris-Sud, Univ. Paris-Saclay, 91192, Gif-sur-Yvette, France vasiliki.reppadcentralesupelec.fr
[3] and FTC [4], [5] techniques for HVAC systems following centralized approaches. However, in many practical applications involving large-scale buildings distributed FTC schemes are more effective since by handling the occurrence of faults locally and exchanging information between neighboring subsystems and local control agents, the delay in fault diagnosis and estimation can be reduced, facilitating the early compensation of faults effects. Nevertheless, there are very few distributed FTC schemes for HVAC systems in the literature (see, for example, [6]).

In previous work [7], the authors have developed an observer-based distributed sensor fault diagnosis scheme with emphasis on the isolation of multiple sensor faults affecting separated building zones (not interconnected) and the electromechanical part of the HVAC system. In [6], an adaptive approximation methodology was used to generate virtual signals of faulty sensors. In more recent work [8], the authors have designed a distributed adaptive estimation scheme for detecting and isolating sensor faults in a HVAC system of a network of interacting building zones, however the fault accommodation (FA) problem was not addressed.

The main contribution of this work is the design of a distributed active FTC scheme for compensating the effects of sensor faults that impact the operation of a HVAC system with multiple, possibly strongly interconnected building zones. By considering the HVAC system as a network of interconnected subsystems (see Section II), we design local monitoring and control agents for every subsystem (see Section III). The local monitoring agent is responsible for diagnosing and estimating local sensor faults (see Sections IIIA-III-B). The main task of each local control agent is to track a corresponding desired signal under both healthy and faulty conditions. Both the local monitoring and control agents are allowed to exchange information with neighboring agents. When sensor faults are isolated by a local monitoring agent, the local and neighboring control agents are reconfigured by using the adaptive estimation of sensor faults transmitted by the neighboring monitoring agents (see Section III-C). The application of proposed methodology to a 7-zone HVAC system is illustrated in Section IV.

\section{PROBLEM FORMULATION}

This section presents the model of a multi-zone HVAC system, which is formulated according to [9]. Fig. 1 depicts the multi-zone HVAC system with the electromechanical part (green box) and the $\mathrm{N}$ building zones, which are interconnected through doors [10]. Let us consider the HVAC system as a network of $N+1$ interconnected subsystems, where the subsystem associated with the water temperature dynamics in the storage tank is denoted by $\Sigma^{s}$, and $\Sigma^{(i)}$ represents 


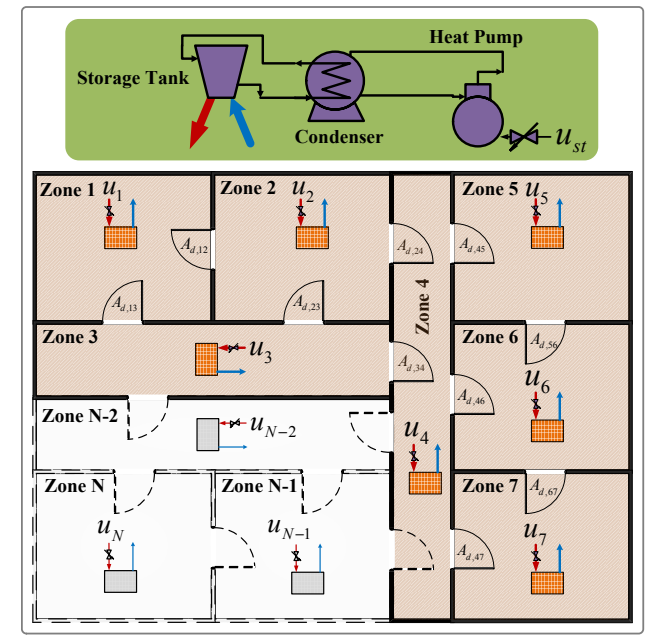

Fig. 1. Illustrative example of a multi-zone HVAC system that consists of the electromechanical part (green box) and the 7 zones (the orange rectangular boxes represent the fan-coil units) .

the subsystem associated with the $i$-th zone temperature dynamics, $i \in \mathcal{N}$, where $\mathcal{N}=\{1, \ldots, N\}$. Based on the thermal mass balance equation presented analytically in [8], the state space form of $\Sigma^{s}$ can be expressed as

$$
\begin{aligned}
\Sigma^{s}: \dot{x}^{s}(t)= & A^{s} x^{s}(t)+g^{s}\left(x^{s}(t), d^{s}(t)\right) u^{s}(t) \\
& +h^{s}\left(x^{s}(t), x(t), u(t)\right)+\eta^{s}\left(d^{s}(t)\right)+r^{s}(t),
\end{aligned}
$$

where $x^{s} \in \mathbb{R}$ and $u^{s} \in \mathbb{R}$ are the state and input of $\Sigma^{s}$ that respectively correspond to the temperature of the water in the storage tank and the controlled normalized energy in the heat pump, while $r^{s} \in \mathbb{R}$ accounts for the modeling uncertainty in temperature dynamics of $\Sigma^{s}$. The term $x$ is the interconnection vector defined as $x \triangleq\left[x^{(1)}, \ldots, x^{(N)}\right]$, where $x^{(i)} \in \mathbb{R}$ represents the state of $\Sigma^{(i)}$ that is the zone air temperature, and $u \triangleq\left[u^{(1)}, \ldots, u^{(N)}\right]$, where $u^{(i)} \in \mathbb{R}$ is the input vector of $\Sigma^{(i)}$ that corresponds to the controlled mass flow rate of water flowing into each fan-coil (see Fig. 1). The variable $d^{s} \in \mathbb{R}^{2}$ represents uncontrollable but known exogeneous inputs, defined as $d^{s} \triangleq\left[d_{1}^{s}, d_{2}^{s}\right]^{\top}$, where $d_{1}^{s}$ is the plenum (duct) temperature and $d_{2}^{s}$ is the source heat temperature to the heat pump, and $\eta^{s}\left(d^{s}\right)=\frac{a_{s t}}{C_{s t}} d_{1}^{s}$. The parameter $A^{s}$ is defined as $A^{s}=-\frac{a_{s t}}{C_{s t}}$. The terms $g^{s}(.) u^{s}$ and $h^{s}($.$) describe the nonlinear local and interconnection$ dynamics of the subsystem $\Sigma^{s}$, respectively, with

$$
\begin{aligned}
g^{s}\left(x^{s}, d^{s}\right) & =\frac{U_{s t, \max }}{C_{s t}}\left(1+p\left(1-\frac{x^{s}(t)-d_{2}^{s}(t)}{\Delta T_{\max }}\right)\right) \\
h^{s}\left(x^{s}, x^{(i)}, u\right) & =\frac{a_{s z}}{C_{s t}} \sum_{i \in \mathcal{N}} U_{i, \max }\left(x^{s}-x^{(i)}\right) u,
\end{aligned}
$$

with $p=\left(P_{\max }-1\right)$, where $P_{\max }$ is the rated maximum value of the performance coefficient of the heat pump, and $\Delta T_{\max }\left({ }^{\circ} \mathrm{C}\right)$ is the maximum temperature difference of the heat pump. The parameter $C_{s t}\left(\mathrm{~kJ} /{ }^{\circ} \mathrm{C}\right)$ is the heat capacity of the storage tank, $U_{i, \max }(\mathrm{kg} / \mathrm{h})$ is the maximum mass flow rate of hot water through the coil placed at $i$-th zone, and $U_{s t, \max }(\mathrm{kJ} / \mathrm{h})$ is the heat pump rated capacity. The coefficients $a_{s z}$ and $a_{s t}$
$\left(\mathrm{kJ} / \mathrm{kg}{ }^{o} \mathrm{C}\right)$ represent the effectiveness of the heating coil and the heat loss of the storage tank due to exterior surfaces, respectively.

Based on [8] and [10], the state space representation of the subsystem $\Sigma^{(i)}, \quad i \in \mathcal{N}$, can be expressed as

$$
\begin{aligned}
\Sigma^{(i)}: \dot{x}^{(i)}(t)= & A^{(i)} x^{(i)}(t)+g^{(i)}\left(x^{s}(t), x^{(i)}(t)\right) u^{(i)}(t)+r^{(i)}(t) \\
& +h^{(i)}\left(x^{(i)}(t), x^{(j)}(t)\right)+\eta^{(i)}\left(d^{(i)}(t)\right),
\end{aligned}
$$

where $r^{(i)} \in \mathbb{R}$ denotes the uncertainty in the temperature dynamics of $\Sigma^{(i)}, \quad i \in \mathcal{N}$. The term $d^{(i)} \in \mathbb{R}^{2}$ is the uncontrollable but known exogenous input vector, defined as $d^{(i)} \triangleq\left[d_{1}^{(i)}, d_{2}^{(i)}\right]^{\top}$, where $d_{1}^{(i)}$ is the temperature of the surface node of the mass wall in the $i$-th zone, $d_{2}^{(i)}$ is the ambient temperature, and $\eta^{(i)}\left(d^{(i)}\right)=\frac{a_{z_{i}}}{C_{z_{i}}} d_{1}^{(i)}-\frac{h A_{d}}{C_{z_{i}}} d_{2}^{(i)}$. The parameter $A^{(i)}$ is defined as $A^{(i)}=\frac{h A_{w_{i}}-a_{z_{i}}}{C_{z_{i}}}-\frac{1}{C_{z_{i}}} \sum_{j \in \mathcal{K}_{i}} a_{z_{i j}}$. Note that the variables $d_{1}^{s}(t), d_{2}^{s}(t), d_{1}^{(i)}(t)$ and $d_{2}^{(i)}(t)$ are usually measured, but, for the sake of simplicity in this work, they are considered known. The terms $g^{(i)}(.) u^{(i)}$ and $h^{(i)}($.$) denote$ the nonlinear interconnection dynamics of the subsystem $\Sigma^{(i)}$ with the subsystem $\Sigma^{s}$ and $\Sigma^{(j)}, j \in \mathcal{K}_{i}$, respectively, where $\mathcal{K}_{i}$ is a set that includes the indices of zones interconnected with the $i$-th zone, and

$$
\begin{aligned}
g^{(i)}\left(x^{s}, x^{(i)}\right)= & \frac{U_{i, \max } a_{s z}}{C_{z_{i}}}\left(x^{s}-x^{(i)}\right), \\
h^{(i)}\left(x^{(i)}, x^{(j)}\right)= & \frac{1}{C_{z_{i}}} \sum_{j \in \mathcal{K}_{i}} a_{z_{i j}} x^{(j)}+\frac{\rho_{a i r} C_{p}}{C_{z_{i}}}\left(\sum_{j \in \mathcal{K}_{i}} \operatorname{sgn}\left(x^{(j)}-x^{(i)}\right)\right. \\
& \left.\times A_{d_{i j}} x^{(i)} \sqrt{2\left(C_{p}-C_{v}\right)\left|x^{(j)}-x^{(i)}\right|}\right),
\end{aligned}
$$

where $A_{d_{i j}}\left(\mathrm{~m}^{2}\right)$ is the area of the door connecting the $i$-th and $j$-th zone, and $C_{z_{i}}\left(\mathrm{~kJ} /{ }^{\circ} \mathrm{C}\right)$ is the air heat capacity of the $i$-th zone. The coefficient $a_{z_{i}}\left(\mathrm{~kJ} / \mathrm{h}{ }^{\circ} \mathrm{C}\right)$ corresponds to the heat loss of $i$-th zone, and $a_{z_{i j}}\left(\mathrm{~kJ} / \mathrm{h}{ }^{\circ} \mathrm{C}\right)$ is the interzone thermal flow coefficient between $i$-th and $j$-th zone. The parameters $C_{p}(\mathrm{~kJ} / \mathrm{kg} \mathrm{K}), C_{v}(\mathrm{~kJ} / \mathrm{kg} \mathrm{K})$, and $\rho_{\text {air }}(\mathrm{kg}$ $/ \mathrm{m}^{3}$ ) represent the specific heat capacity of air at constant pressure, the specific heat capacity of air at constant volume, and the air density, respectively, and $h\left(\mathrm{~W} / \mathrm{m}^{2}{ }^{o} \mathrm{C}\right)$ is the heat transfer coefficient and $A_{w_{i}}\left(\mathrm{~m}^{2}\right)$ is the surface area of the mass wall of the $i$-th zone. Note that the set $\mathcal{K}_{i}$ is defined as $\mathcal{K}_{i}=\left\{j \in \mathcal{N} \backslash\{i\}: a_{z_{i j}} \neq 0\right\}$.

The subsystems $\Sigma^{s}$ and $\Sigma^{(i)}$ are monitored and controlled using the sensors $\mathcal{S}^{s}$ and $\mathcal{S}^{(i)}$, respectively, which are characterized by the outputs $y^{s} \in \mathbb{R}$ and $y^{(i)} \in \mathbb{R}$; i.e.,

$$
\begin{gathered}
\mathcal{S}^{s}: y^{s}(t)=x^{s}(t)+n^{s}(t)+f^{s}(t), \\
\mathcal{S}^{(i)}: y^{(i)}(t)=x^{(i)}(t)+n^{(i)}(t)+f^{(i)}(t),
\end{gathered}
$$

where $n^{s}$ and $n^{(i)}$ represent the noise corrupting the sensor measurements, respectively, and $f^{s}$ and $f^{(i)}$ denote permanent sensor faults, modeled as in [7].

The objective of this work is to design an active, distributed fault tolerant control methodology that detects and isolates sensor faults that may affect one or more subsystems, and compensates the fault effects in the distributed control 
scheme that generates the signals $u^{s}$ and $u^{(i)}, i \in\{1, \ldots, N\}$.

\section{Distributed Sensor Fault Tolerant Scheme}

The backbone of the distributed sensor fault tolerant control (FTC) scheme is the design of two agents dedicated to each of the interconnected HVAC subsystems. Particularly, the monitoring agents, denoted by $\mathcal{M}^{s}$ and $\mathcal{M}^{(i)}$, are responsible for detecting and isolating sensor faults in subsystems $\Sigma^{s}$ and $\Sigma^{(i)}, i \in \mathcal{N}$, respectively, and estimating the local sensor fault after isolation. The control agents $\mathcal{C}^{s}$ and $\mathcal{C}^{(i)}$ are responsible for tracking the reference signals for subsystems $\Sigma^{s}$ and $\Sigma^{(i)}$ respectively, under healthy and faulty conditions using the sensor fault estimations provided by the agents $\mathcal{M}^{s}$ and $\mathcal{M}^{(i)}$. The monitoring and control agents are allowed to exchange information with neighboring agents. In this work, the emphasis is on sensor faults, so we assume that there are no imperfections in the communication between the control and monitoring agents.

\section{A. Distributed Adaptive Estimation Scheme}

The decision of the monitoring agents $\mathcal{M}^{s}$ and $\mathcal{M}^{(i)}$ on the occurrence of sensor faults is obtained by checking the satisfaction of analytical redundancy relations (ARRs) of residuals and adaptive thresholds, as presented next.

1) Residual Generation: The residual generated by the agents $\mathcal{M}^{s}$ and $\mathcal{M}^{(i)}$ is respectively defined as $\varepsilon_{y}^{s}(t)=y^{s}(t)$ $\hat{x}^{s}(t)$ and $\varepsilon_{y}^{(i)}(t)=y^{(i)}(t)-\hat{x}^{(i)}(t)$, where $\hat{x}^{s}$ and $\hat{x}^{(i)}$ are the estimations of $x^{s}$ and $x^{(i)}$, respectively, computed based on the following nonlinear adaptive estimation models

$$
\begin{aligned}
\dot{\hat{x}}^{s}(t)= & A^{s} \hat{x}^{s}(t)+g^{s}\left(y^{s}(t)-\hat{f}^{s}(t), d^{s}(t)\right) u^{s}(t) \\
& +h^{s}\left(y^{s}(t)-\hat{f}^{s}(t), y(t), u(t)\right)+\eta^{s}\left(d^{s}(t)\right) \\
& +L^{s}\left(y^{s}(t)-\hat{x}^{s}(t)-\hat{f}^{s}(t)\right)+\Omega^{s}(t) \hat{\hat{f}}^{s}(t), \\
\dot{\Omega}^{s}(t)= & A_{L}^{s} \Omega^{s}(t)-L^{s}+\frac{U_{s t, \max }\left(P_{\max }-1\right)}{C_{s t} \Delta T_{\max }} u^{s}(t) \\
& +\frac{a_{s z}}{C_{s t}} \sum_{i \in \mathcal{N}} U_{i, \max } u^{(i)}(t), \\
\dot{\hat{f}}^{s}(t)= & \gamma^{s}\left(\Omega^{s}(t)+1\right) \mathcal{D}^{s}\left[\xi_{y}^{s}(t)\right], \\
\xi_{y}^{s}(t)= & y^{s}(t)-\hat{x}^{s}(t)-\hat{f}^{s}(t), \\
\mathcal{D}^{s}\left[\xi_{y}^{s}(t)\right]= & \left\{\begin{array}{cc}
0, & \text { if } I^{s}(t)=0 \\
\xi_{y}^{s}(t), & \text { if } I^{s}(t)=1
\end{array}\right.
\end{aligned}
$$

and

$$
\begin{aligned}
\dot{\hat{x}}^{(i)}(t)= & A^{(i)} \hat{x}^{(i)}(t)+g^{(i)}\left(y^{s}(t), y^{(i)}(t)-\hat{f}^{(i)}(t)\right) u^{(i)}(t) \\
& +h^{(i)}\left(y^{(i)}(t)-\hat{f}^{(i)}(t), y^{(j)}(t)\right)+\eta^{(i)}\left(d^{(i)}(t)\right) \\
& +L^{(i)}\left(y^{(i)}(t)-\hat{x}^{(i)}(t)-\hat{f}^{(i)}(t)\right) \\
& +\Omega^{(i)}(t) \hat{f}^{(i)}(t), \\
\dot{\Omega}^{(i)}(t)= & A_{L}^{(i)} \Omega^{(i)}(t)-L^{(i)}-\frac{U_{i, \max } a_{s z}}{C_{z_{i}}} u^{(i)}(t), \\
\hat{f}^{(i)}(t)= & \gamma^{(i)}\left(\Omega^{(i)}(t)+1\right) \mathcal{D}^{(i)}\left[\xi_{y}^{(i)}(t)\right], \\
\xi_{y}^{(i)}(t)= & y^{(i)}(t)-\hat{x}^{(i)}(t)-\hat{f}^{(i)}(t), \\
\mathcal{D}^{(i)}\left[\xi_{y}^{(i)}(t)\right]= & \left\{\begin{array}{cc}
0, & \text { if } I^{(i)}(t)=0 \\
\xi_{y}^{(i)}(t), & \text { if } I^{(i)}(t)=1
\end{array}\right.
\end{aligned}
$$

where $y \triangleq\left[y^{(1)}, \ldots, y^{(N)}\right]^{\top}$ and $L^{s}, L^{(i)}$ are the estimation gains, such that $A_{L}^{s} \triangleq A^{s}-L^{s}, A_{L}^{(i)} \triangleq A^{(i)}-L^{(i)}$ are stable. The terms $\hat{f}^{s}, \hat{f}^{(i)}$ are the estimations of the faults $f^{s}, f^{(i)}$, respectively with $\hat{f}^{s}\left(t_{I}^{s}\right)=0$ and $\hat{f}^{(i)}\left(t_{I}^{(i)}\right)=0$, and $\Omega^{s}$, $\Omega^{(i)}$ are filtering terms necessary for ensuring the stability property of the adaptive nonlinear estimation schemes [11] with $\Omega^{s}\left(t_{I}^{s}\right)=0$ and $\Omega^{(i)}\left(t_{I}^{(i)}\right)=0$, where $t_{I}^{s}$ and $t_{I}^{(i)}$ are the isolation times defined as $t_{I}^{s} \triangleq \min \left\{t \geq 0: I^{s}(t)=1\right\}, t_{I}^{(i)} \triangleq$ $\min \left\{t \geq 0: I^{(i)}(t)=1\right\}$, with $I^{s}, I^{(i)}$ being the corresponding isolation signals (see Section III-B).

2) Computation of Adaptive Thresholds: The adaptive thresholds $\bar{\varepsilon}_{y}^{s}$ and $\bar{\varepsilon}_{y}^{(i)}$ are designed to bound the corresponding residuals under healthy conditions (i.e., $f^{s}=0, f^{(i)}=0$ for all $i \in \mathfrak{N}$ ) taking into account the following assumption:

Assumption 1: The measurement noise $n^{s}, n^{(i)}$ and the modeling uncertainty $r^{s}, r^{(i)}$ are uniformly bounded by known positive constants $\bar{n}^{s}, \bar{n}^{(i)}, \bar{r}^{s}$ and $\bar{r}^{(i)}$ such that $\left|n^{s}(t)\right| \leq \bar{n}^{s},\left|n^{(i)}(t)\right| \leq \bar{n}^{(i)},\left|r^{s}(t)\right| \leq \bar{r}^{s}$ and $\left|r^{(i)}(t)\right| \leq \bar{r}^{(i)}$, respectively.

Particularly, $\left|\varepsilon_{y}^{s}(t)\right| \leq \bar{\varepsilon}^{s}(t)$ and $\left|\varepsilon_{y}^{(i)}(t)\right| \leq \bar{\varepsilon}_{y}^{(i)}(t)$ with

$$
\begin{aligned}
\mathcal{E}_{y}^{s}(t)= & y^{s}(t)-\hat{x}^{s}(t) \\
= & e^{A_{L}^{s} t} \varepsilon_{x}^{s}(0)+n^{s}(t)+\int_{0}^{t} e^{A_{L}^{s}(t-\tau)}\left(r^{s}(\tau)-L^{s} n^{s}(\tau)\right. \\
& +\left(g^{s}\left(x^{s}(\tau), d^{s}(\tau)\right)-g^{s}\left(y^{s}(\tau), d^{s}(\tau)\right)\right) u^{s}(\tau) \\
& \left.+h^{s}\left(x^{s}(\tau), x(\tau), u(\tau)\right)-h^{s}\left(y^{s}(\tau), y(\tau), u(\tau)\right)\right) d \tau .
\end{aligned}
$$

and

$$
\begin{aligned}
\varepsilon_{y}^{(i)}(t) & =y^{(i)}(t)-\hat{x}^{(i)}(t) \\
& =e^{A_{L}^{(i)} t} \varepsilon_{x}^{(i)}(0)+n^{(i)}(t)+\int_{0}^{t} e^{A_{L}^{(i)}(t-\tau)}\left(r^{(i)}(\tau)-L^{(i)} n^{(i)}(\tau)\right. \\
& +\left(g^{(i)}\left(x^{s}(\tau), x^{(i)}(\tau)\right)-g^{(i)}\left(y^{s}(\tau), y^{(i)}(\tau)\right)\right) u^{(i)}(\tau) \\
& \left.+h^{(i)}\left(x^{(i)}(\tau), x^{(j)}(\tau)\right)-h^{(i)}\left(y^{(i)}(\tau), y^{(j)}(\tau)\right)\right) d \tau .
\end{aligned}
$$

After some mathematical manipulations, the adaptive threshold of $\mathcal{M}^{s}$ is defined as

$$
\begin{aligned}
\bar{\varepsilon}_{y}^{s}(t)= & \rho^{s} e^{-\lambda^{s} t} \bar{x}^{s}+\int_{0}^{t} \rho^{s} e^{-\lambda^{s}(t-\tau)}\left(\left|\frac{U_{s t \max }\left(P_{\max }-1\right)}{C_{s t} \Delta T_{\max }}\right| \bar{n}^{s}\left|u^{s}(\tau)\right|\right. \\
& \left.+\left|L^{s}\right| \bar{n}^{s}+\frac{a_{s t}}{C_{s t}} \sum_{i \in \mathcal{N}} U_{i, \max }\left(\bar{n}^{s}+\bar{n}^{(i)}\right)\left|u^{(i)}(\tau)\right|+\bar{r}^{s}\right) d \tau+\bar{n}^{s},
\end{aligned}
$$

and the adaptive threshold $\bar{\varepsilon}_{y}^{(i)}$ of $\mathcal{M}^{(i)}$ is defined as

$$
\begin{aligned}
\bar{\varepsilon}_{y}^{(i)}(t) & =\rho^{(i)} e^{-\lambda^{(i)} t} \bar{x}^{(i)}+\bar{n}^{(i)}+\int_{0}^{t} \rho^{(i)} e^{-\lambda^{(i)}(t-\tau)}\left(\left|L^{(i)}\right| \bar{n}^{(i)}+\bar{r}^{(i)}\right. \\
& +\frac{U_{i, m a x} a_{s z}}{C_{z_{i}}}\left(\bar{n}^{(i)}+\bar{n}^{s}\right)\left|u^{(i)}(\tau)\right|+\frac{1}{C_{z_{i}}} \sum_{j \in \mathcal{K}_{i}} a_{z_{i j}}\left(\bar{n}^{(i)}+\bar{n}^{(j)}\right) \\
& \left.+\frac{\rho_{a i r} C_{p}}{C_{z_{i}}} \sqrt{2\left|C_{p}-C_{v}\right|} \sum_{j \in \mathcal{K}_{i}} A_{d_{i j}} \bar{h}^{(i)}\left(y^{(i)}(\tau), y^{(j)}(\tau)\right)\right) d \tau
\end{aligned}
$$

where $\bar{x}^{s}, \bar{x}^{(i)}$ are known bounds such that $\left|x^{s}(0)\right| \leq \bar{x}^{s}$ and $\left|x^{(i)}(0)\right| \leq \bar{x}^{(i)}$, and $\rho^{s}>0, \quad \lambda^{s}>0, \quad \rho^{(i)}>$ $0, \quad \lambda^{(i)}>0$ are respectively selected such that $\left|e^{A_{L}^{s} t}\right| \leq \rho^{s} e^{-\lambda^{s} t}$ and $\left|e^{A_{L}^{(i)}}\right| \leq \rho^{(i)} e^{-\lambda^{(i)} t}$, for all $t$. Note that the term $\bar{h}^{(i)}\left(y^{(i)}, y^{(j)}\right)$ is defined as $\bar{h}^{(i)}\left(y^{(i)}, y^{(j)}\right)=\left(\left|y^{(i)}\right|+\bar{n}^{(i)}\right) \sqrt{\bar{n}^{(j)}+\bar{n}^{(i)}}$, if $y^{(i)}=y^{(j)}$ and $\bar{h}^{(i)}\left(y^{(i)}, y^{(j)}\right)=\max \left(\left|y^{(i)}\right| \sqrt{\left|y^{(j)}-y^{(i)}\right|},\left(\bar{n}^{(i)} \frac{\left|2 y^{(j)}-3 y^{(i)}\right|}{2\left|y^{(j)}-y^{(i)}\right|}+\right.\right.$ 
$\left.\left.\bar{n}^{(j)} \frac{\left|y^{(i)}\right|}{2\left|y^{(j)}-y^{(i)}\right|}+\bar{h}_{e}^{(i)}\right)\right)$, otherwise, with $\bar{h}_{e}^{(i)}$ being a constant bound on the linearization error of $h^{(i)}$ around $x^{(i)}=y^{(i)}$ and $x^{(j)}=y^{(j)}$ due to the higher-order terms of the Taylor series expansion of (6).

3) Sensor Fault Detection Decision Logic: The sensor fault detection decision logic implemented in $\mathcal{M}^{s}$ and $\mathcal{M}^{(i)}$ relies on ARRs of the residuals and adaptive thresholds, which are respectively determined as

$$
\mathcal{E}^{s}:\left|\varepsilon_{y}^{s}(t)\right| \leq \bar{\varepsilon}_{y}^{s}(t), \quad \mathcal{E}^{(i)}:\left|\mathcal{E}_{y}^{(i)}(t)\right| \leq \bar{\varepsilon}_{y}^{(i)}(t) .
$$

The agent $\mathcal{M}^{s}$ (correspondingly for $\mathcal{M}^{(i)}$ ) infers the presence of sensor faults at the first time instant that $\mathcal{E}^{s}$ is not satisfied (correspondingly for $\mathcal{E}^{(i)}$ ). Note that the ARR $\mathcal{E}^{s}$ is sensitive to faults in sensors $\mathcal{S}^{s}$ and $\mathcal{S}^{(i)}, i \in \mathcal{N}$, while $\mathcal{E}^{(i)}$ is sensitive to faults that may affect $\mathcal{S}^{s}, \mathcal{S}^{(i)}$ and $\mathcal{S}^{(j)}$, $j \in \mathcal{K}_{i}$. The outputs of the agent $\mathcal{M}^{s}$ and $\mathcal{M}^{(i)}$ are the boolean decision signals $D^{s}$ and $D^{(i)}$, defined as

$$
D^{s}(t)=\left\{\begin{array}{c}
0, t<t_{D}^{s} \\
1, t \geq t_{D}^{s}
\end{array} \quad, \quad D^{(i)}(t)=\left\{\begin{array}{c}
0, t<t_{D}^{(i)} \\
1, t \geq t_{D}^{(i)}
\end{array},\right.\right.
$$

where $t_{D}^{s} \triangleq \inf \left\{t \geq 0:\left|\varepsilon_{y}^{s}(t)\right|>\bar{\varepsilon}_{y}^{s}(t)\right\}$ and $t_{D}^{(i)} \triangleq \inf \{t \geq 0$ : $\left.\left|\mathcal{E}_{y}^{(i)}(t)\right|>\bar{\varepsilon}_{y}^{(i)}(t)\right\}$. If $\mathcal{E}^{s}$ and $\mathcal{E}^{(i)}$ are always satisfied, then $t_{D}^{s} \rightarrow \infty$ and $t_{D}^{(i)} \rightarrow \infty$.

\section{B. Distributed Sensor Fault Isolation Decision Logic}

The sensor fault isolation process of the agent $\mathcal{M}^{s}$ initiates the isolation process when it detects the presence of sensor faults, or when at least one of the $N$ agents $\mathcal{M}^{(i)}, i \in \mathcal{N}$ does it, while the agent $\mathcal{M}^{(i)}$ starts the isolation when the agent itself, or at least one of the neighboring agents $\mathcal{M}^{s}$ and $\mathcal{M}^{(j)}, j \in \mathcal{K}_{i}$ detects the presence of sensor faults.

The distributed isolation procedure applied by a monitoring agent involves the comparison of the observed pattern of sensor faults that may affect the neighborhood of the agent to a number of theoretical patterns, represented by the columns of a sensor fault signature matrix. In the case of the agent $\mathcal{M}^{s}$, the observed pattern of sensor faults, denoted by $\Phi^{s}(t) \epsilon$ $[0,1]^{N+1}$, where $[0,1]^{N+1}$ denotes a binary vector of $N+1$ length, and defined as $\Phi^{s}(t)=\left[D^{s}, D^{(1)}, \ldots, D^{(N)}\right]$, where $D^{s}, D^{(i)}$ are defined in (24). Note that $D^{(i)}$ is transmitted to $\mathcal{M}^{s}$ by the agent $\mathcal{M}^{(i)}$ for all $i \in \mathcal{N}$. The sensor fault signature matrix consists of $N+1$ rows, which correspond to the set of ARRs $\left\{\mathcal{E}^{s}, \mathcal{E}^{(1)}, \ldots, \mathcal{E}^{(N)}\right\}$, and $N_{c}=2^{N+1}-1$ columns that correspond to all possible sensor fault combinations that may affect the building zones and the storage tank, where the $k$-th combination is indicated by $\mathcal{F}_{c_{k}}^{s}, k \epsilon$ $\left\{1, \ldots, N_{c}\right\}$. The $k$-th column corresponds to the theoretical pattern, denoted by $F_{k}^{s}$ and defined as $F_{k}^{s}=\left[F_{k 1}^{s}, \ldots, F_{k N+1}^{s}\right]^{\top}$. Taking into account the 7-zone HVAC system shown in Fig. 1 , the sensor fault signature matrix $F^{s}$ is comprised of 8 rows and 255 columns. Table I illustrates a part of $F^{s}$, assuming the occurrence of 8 single sensor faults, and one possible combination of a combination of two simultaneous sensor faults. The assignment of the values to the elements $F_{q k}^{s}$ is realized such that the sensitivity of every ARRs to local and propagated sensor faults is distinguished [12]. For example, $f^{(1)}$ is involved in $\mathcal{E}^{(1)}$ since it is a fault that may affect the local sensor $\mathcal{S}^{(1)}$, while it is also involved in $\mathcal{E}^{s}$, since it may propagated through the exchange of information between the agent $\mathcal{M}^{(i)}$ and $\mathcal{M}^{s}$. Hence, the assignment $F_{22}^{s}=1$ implies that $f^{(1)}$ necessarily discloses its occurrence by provoking the violation of $\mathcal{E}^{(1)}$, while $F_{12}^{s}=*$ implies that $f^{(1)}$ may justify the violation of $\mathcal{E}^{s}$, but $\mathcal{E}^{s}$ may be satisfied in spite of its occurrence. On the other hand, $F_{52}^{s}=0$, since $f^{(1)}$ is not involved in $\mathcal{E}^{(5)}$. In the case of the

TABLE I

SENSOR FAULT SIGNATURE MATRIX $F^{s}$

\begin{tabular}{|c|c|c|c|c|c|c|c|c|c|}
\cline { 2 - 9 } \multicolumn{1}{c|}{} & $f^{s}$ & $f^{(1)}$ & $f^{(2)}$ & $f^{(3)}$ & $f^{(4)}$ & $f^{(5)}$ & $f^{(6)}$ & $f^{(7)}$ & $\left\{f^{s}, f^{(1)}\right\}$ \\
\hline $\mathcal{E}^{s}$ & 1 & $*$ & $*$ & $*$ & $*$ & $*$ & $*$ & $*$ & 1 \\
\hline $\mathcal{E}^{(1)}$ & $*$ & 1 & $*$ & $*$ & 0 & 0 & 0 & 0 & 1 \\
\hline $\mathcal{E}^{(2)}$ & $*$ & $*$ & 1 & $*$ & $*$ & 0 & 0 & 0 & $*$ \\
\hline $\mathcal{E}^{(3)}$ & $*$ & $*$ & $*$ & 1 & $*$ & 0 & 0 & 0 & $*$ \\
\hline $\mathcal{E}^{(4)}$ & $*$ & 0 & $*$ & $*$ & 1 & $*$ & $*$ & $*$ & $*$ \\
\hline $\mathcal{E}^{(5)}$ & $*$ & 0 & 0 & 0 & $*$ & 1 & $*$ & 0 & $*$ \\
\hline $\mathcal{E}^{(6)}$ & $*$ & 0 & 0 & 0 & $*$ & $*$ & 1 & $*$ & $*$ \\
\hline $\mathcal{E}^{(7)}$ & $*$ & 0 & 0 & 0 & $*$ & 0 & $*$ & 1 & $*$ \\
\hline
\end{tabular}

agent $\mathcal{M}^{(i)}$, the observed pattern of sensor faults, denoted by $\Phi^{(i)}(t) \in[0,1]^{\left|\mathcal{K}_{i}\right|+2}$, is a vector made up the decisions $D^{s}, D^{(i)}$ and $D^{(j)}$ for all $j \in \mathcal{K}_{i}$. The sensor fault signature matrix $F^{(i)}$ consists of $\left|\mathcal{K}_{i}\right|+2$ rows, which correspond to the set of ARRs $\left\{\mathcal{E}^{s}, \mathcal{E}^{(i)}\right\} \bigcup_{j \in \mathcal{K}_{i}}\left\{\mathcal{E}^{(j)}\right\}$, and $N_{c}^{(i)}=2^{\left|\mathcal{K}_{i}\right|+2}-1$ columns. The $k$-th column corresponds to the theoretical pattern, denoted by $F_{k}^{(i)}$. For example, $F^{(1)}$ is structured as in the shaded area of Table I, taking into account 4 single sensor faults and a combination of two simultaneous sensor faults.

The outcome of the comparison of the observed fault pattern $\Phi^{s}$ to the $N_{c}$ theoretical fault patterns $F_{k}^{s}, k \in\left\{1, \ldots, N_{c}\right\}$, and the observed pattern $\Phi^{(i)}$ to the $N_{c}^{(i)}$ theoretical patterns $F_{q}^{(i)}, q \in\left\{1, \ldots, N_{c}^{(i)}\right\}$ is the diagnosis sets $\Upsilon^{s}(t)$ and $\Upsilon^{(i)}(t)$, which are determined as

$$
\Upsilon^{s}(t)=\left\{\mathcal{F}_{c_{i}}^{s}: i \in \mathcal{I}_{\Upsilon}^{s}(t)\right\}, \Upsilon^{(i)}(t)=\left\{\mathcal{F}_{c_{i}}^{(i)}: i \in \mathcal{I}_{\Upsilon}^{(i)}(t)\right\},
$$

with $\mathcal{I}_{\Upsilon}^{s}(t)=\left\{k: F_{k}^{s}=\Phi^{s}(t), k \in\left\{1, \ldots, N_{c}\right\}\right\}$ and $\mathcal{I}_{\Upsilon}^{(i)}(t)=$ $\left\{k: F_{k}^{(i)}=\Phi^{(i)}(t), k \in\left\{1, \ldots, N_{c}^{(i)}\right\}\right\}$. The isolation signals that activate the estimation of local sensor faults in (11)-(13) and (16)-(18), are defined as

$I^{s}(t)=\left\{\begin{array}{l}1, \text { if } f^{s} \in \Upsilon^{s}(t) \\ 0, \text { otherwise }\end{array}, I^{(i)}(t)=\left\{\begin{array}{l}1, \text { if } f^{(i)} \in \Upsilon^{(i)}(t) \\ 0, \text { otherwise }\end{array}\right.\right.$

Additionally, the isolation signal related to the propagated sensor faults $f^{(i)}, i \in \mathcal{N}$, generated by the agent $\mathcal{M}^{s}$ and used in the distributed FA control scheme, is defined as

$$
I^{s, i}(t)=\left\{\begin{array}{l}
1, \text { if } f^{(i)} \in \Upsilon^{s}(t) \\
0, \text { otherwise }
\end{array}\right.
$$

while the isolation signal related to the propagated sensor 
faults $f^{s}$ and $f^{(j)}, j \in \mathcal{K}_{i}$, which is generated by the agent $\mathcal{M}^{(i)}$ and used in the distributed FA control scheme, are defined as

$I^{(i, s)}(t)=\left\{\begin{array}{l}1, \text { if } f^{s} \in \Upsilon^{(i)}(t) \\ 0, \text { otherwise }\end{array}, I^{(i, j)}(t)=\left\{\begin{array}{l}1, \text { if } f^{(j)} \in \Upsilon^{(i)}(t) \\ 0, \text { otherwise }\end{array}\right.\right.$

\section{Distributed Fault Accommodation Control Scheme}

The control agents denoted by $\mathcal{C}^{s}$ and $\mathcal{C}^{(i)}, i \in \mathcal{N}$ are designed such that: (i) under healthy conditions, the nominal distributed control scheme can track the local differentiable reference temperature signals, denoted by $y_{\text {ref }}^{s}$ and $y_{\text {ref }}^{(i)}$, (ii) under faulty conditions, the distributed control scheme is modified based on the isolation signals defined in (26)-(28) in order to compensate the effects of sensor faults by using the adaptive fault estimations generated according to (11)(13) and (16)-(18), and i.e.,

$$
\begin{gathered}
\mathcal{C}^{s}: u^{s}(t)=\chi^{s}\left(y_{v}^{s}(t), y_{v}(t), u(t), y_{\text {ref }}^{s}, \dot{y}_{\text {ref }}^{s}\right), \\
\mathcal{C}^{(i)}: u^{(i)}(t)=\chi^{(i)}\left(y_{v}^{(i)}(t), y_{v}^{(i, s)}(t), y_{v}^{\mathcal{K}_{i}}(t), y_{\text {ref }}^{(i)}, \dot{y}_{\text {ref }}^{(i)}\right),
\end{gathered}
$$

where

$$
\begin{aligned}
y_{v}^{s}(t) & =y^{s}(t)-\mathcal{D}^{s}\left[\hat{f}^{s}(t)\right], \\
y_{v}(t) & =y(t)-\left[\mathcal{D}^{s, 1}\left[\hat{f}^{(1)}(t)\right], \ldots, \mathcal{D}^{s, N}\left[\hat{f}^{(N)}(t)\right]\right], \\
y_{v}^{(i)}(t) & =y^{(i)}(t)-\mathcal{D}^{(i)}\left[\hat{f}^{(i)}(t)\right], \\
y_{v}^{(i, s)}(t) & =y^{s}(t)-\mathcal{D}^{(i, s)}\left[\hat{f}^{s}(t)\right],
\end{aligned}
$$

and $y_{v}^{\mathcal{K}_{i}}(t)$ is a vector made up of $\left|\mathcal{K}_{i}\right|$ elements $y^{(j)}-$ $\mathcal{D}^{(i, j)}\left[\hat{f}^{(j)}(t)\right], j \in \mathcal{K}_{i}$. The terms $\mathcal{D}^{s}[\cdot]$ and $\mathcal{D}^{(i)}[\cdot]$ are deadzone operators defined similarly to (13) and (18), that is $\mathcal{D}^{s}\left[\hat{f}^{s}(t)\right]=\hat{f}^{s}(t)$ if $I^{s}(t)=1$, and $\mathcal{D}^{s}\left[\hat{f}^{s}(t)\right]=0$ otherwise (correspondingly for $\mathcal{D}^{(i)}[\cdot], \mathcal{D}^{s, i}[\cdot], \mathcal{D}^{(i, s)}[\cdot]$ and $\mathcal{D}^{(i, j)}[\cdot]$ ). Based on the design of the distributed FA scheme, every time that sensor faults are isolated in the neighborhood of $\Sigma^{(i)}$, its associated control agent $\mathcal{C}^{(i)}$ (correspondingly for $\mathcal{C}^{s}$ ), and the control agents of the neighboring subsystems, that is $\mathcal{C}^{s}$ and $\mathcal{C}^{(j)}, j \in \mathcal{K}$ are accommodated to the isolated sensor faults by using their estimations .

\section{Simulation Results}

In this section, we illustrate the application of the distributed adaptive FTC methodology to a 7-zone HVAC system where the architectural arrangement of the 7 zones is presented by the shaded area in Fig. 1. We consider eight subsystems $\left\{\Sigma^{s}, \Sigma^{(1)}, \ldots, \Sigma^{(7)}\right\}$, with the interconnections between the zones, defined by the sets $\mathcal{K}_{1}=\{2,3\}$, $\mathcal{K}_{2}=\{1,3,4\}, \mathcal{K}_{3}=\{1,2,4\}, \mathcal{K}_{4}=\{2,3,5,6,7\}, \mathcal{K}_{5}=\{4,6\}$, $\mathcal{K}_{6}=\{4,5,7\}, \mathcal{K}_{7}=\{4,6\}$. The parameters of the subsystems (1) and (4) are defined in [8], where some additional parameters are defined as follows: $A_{w_{i}}=120, h=8.29, A_{d_{i j}}=2.60$, $i \in \mathcal{N}=\{1, \ldots, 7\}, j \in \mathcal{K}_{i}$. It is assumed that the exogenous uncontrollable signals are constant, defined as follows: $d_{1}^{s}=10^{\circ} C, d_{2}^{s}=5^{\circ} C, d_{1}^{(i)}=5^{\circ} C, d_{2}^{(i)}=10^{\circ} C, i \in \mathcal{N}$. The modeling uncertainty in each subsystem $r^{s}=5 \% d_{1}^{s} \sin (0.1 t)$ and $r^{(i)}=5 \% d_{1}^{(i)} \sin (0.1 t), i \in \mathcal{N}$. For simulation purposes, the noise corrupting the sensor output is defined as: $\bar{n}^{s}=$ $3 \% Y^{s}$ and $\bar{n}^{(i)}=3 \% Y^{(i)}$, where $Y^{s}$ and $Y^{(i)}$ are the steady state value of sensor measurements $y^{s}$ and $y^{(i)}$, respectively, $i \in \mathcal{N}$, under healthy conditions. The design constants for the monitoring agents are: $L^{s}=10$ and $L^{(i)}=1, \rho^{s}=\rho^{(i)}=1.3$, $\lambda^{s}=30, \lambda^{(i)}=6, \gamma^{s}=8, \gamma^{(i)}=5$. In this example, we simulated the following multiple sensor fault scenario: two bias abrupt faults affect sensors $\mathcal{S}^{(1)}$ and $\mathcal{S}^{(7)}$ at $t_{f}^{(1)}=t_{f}^{(7)}=20 \mathrm{~h}$. The faults are modeled as $f^{(1)}(t)=-20 \% Y^{(1)}\left(1-e^{-10^{4}(t-20)}\right)$ and $f^{(7)}(t)=-20 \% Y^{(7)}\left(1-e^{-10^{4}(t-20)}\right)$ [7]. For comparison purposes, we performed simulations with and without using the proposed distributed FA control scheme described by (29)-(30). The desired values of the temperatures are set up as follows: $y_{r e f}^{s}=55^{\circ} \mathrm{C}$ and $y_{\text {ref }}^{(i)}=24{ }^{\circ} \mathrm{C}, i \in \mathcal{N}$.

Fig. 2 shows the residuals, adaptive thresholds and the detection signals of the monitoring agents $\mathcal{M}^{(1)}, \mathcal{M}^{(7)}$, while the rest of the monitoring agents (i.e., $\mathcal{M}^{s}, \mathcal{M}^{(2)}, \ldots, \mathcal{M}^{(6)}$ ) are not presented since their ARRs are not violated for all $t$. At the time instant $t_{D}^{(7)}=20 \mathrm{~h}$, a fault is detected by $\mathcal{M}^{(7)}$ which initiates the isolation procedure in the monitoring agent $\mathcal{M}^{(7)}$ and its neighbouring agents $\mathcal{M}^{s}, \mathcal{M}^{(4)}$ and $\mathcal{M}^{(6)}$. In the case of $\mathcal{M}^{s}$, the observed pattern $\Phi^{s}$ equal to $\Phi^{s}=[0,0,0,0,0,0,0,1]$, which is compared to the theoretical patterns $F_{k}^{s}$ for all $k \in\{1, \ldots, 255\}$, leading to $\Upsilon^{s}=\left\{f^{(7)}\right\}$ and $I^{s}=I^{s, 1}=\cdots=I^{s, 6}=0$ and $I^{s, 7}=1$. A similar isolation process is realized by $\mathcal{M}^{(4)}, \mathcal{M}^{(6)}, \mathcal{M}^{(7)}$, which led to $\Upsilon^{(4)}=\Upsilon^{(6)}=\Upsilon^{(7)}=\left\{f^{(7)}\right\}$. Based on these diagnosis sets the non-zero isolation signals are $I^{(4,7)}, I^{(6,7)}$ and $I^{(7)}$. The dead-zone operator $\mathcal{D}^{(7)}[$.$] activated the estimation for the$ sensor fault $\hat{f}^{(7)}$ executed by agent $\mathcal{M}^{(7)}$. The local control agents that are reconfigured to the isolated fault $f^{(7)}$ using its estimation transmitted by the agent $\mathcal{M}^{(7)}$ are $\mathcal{C}^{s}, \mathcal{C}^{(4)}, \mathcal{C}^{(6)}$, and $\mathcal{C}^{(7)}$. Similarly, at the time instant $t_{D}^{(1)}=20.05 \mathrm{~h}$, a fault

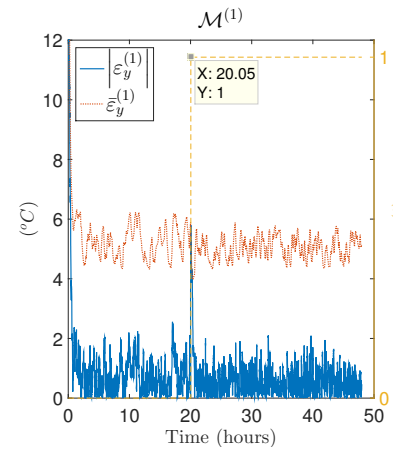

(a)

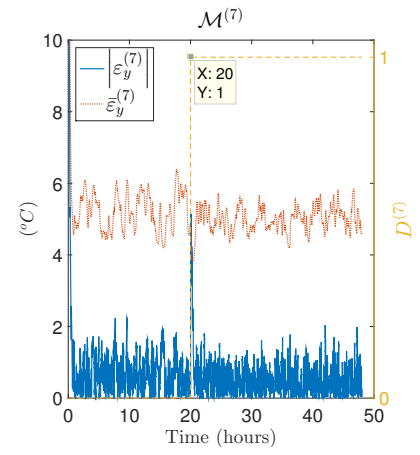

(b)
Fig. 2. Residuals $\varepsilon^{(1)}, \varepsilon^{(7)}$ (blue solid line), adaptive thresholds $\bar{\varepsilon}^{(1)}$, $\bar{\varepsilon}^{(7)}$ (red dotted line) and detection signals $D^{(1)}, D^{(7)}$ (yellow dashed line) of the monitoring agents $\mathcal{M}^{(1)}, \mathcal{M}^{(7)}$

is detected by $\mathcal{M}^{(1)}$ which initiates the isolation procedure locally and its neighboring monitoring agents $\mathcal{M}^{s}, \mathcal{M}^{(2)}$ and $\mathcal{M}^{(3)}$. By applying the distributed isolation process shown in Section III-B, the new non-zero isolation signals are generated: $I^{s, 1}, I^{(2,1)}$, and $I^{(3,1)}$. The dead-zone operator $\mathcal{D}^{(1)}$ [.] enables the estimation for the sensor fault $\hat{f}^{(1)}$ in $\mathcal{M}^{(1)}$, followed by the accommodation of control agents $\mathcal{C}^{s}$, $\mathcal{C}^{(2)}, \mathcal{C}^{(3)}$, and $\mathcal{C}^{(1)}$ to the new isolated sensor faults. Note 
that $\mathcal{C}^{s}$ has been accommodated twice.

Fig. 3 presents the response of the temperatures, controlled either by the nominal control scheme or by the distributed FTC scheme. The faults occur in $\mathcal{S}^{(1)}, \mathcal{S}^{(7)}$, significantly effect the temperature dynamics of the local subsystems $\Sigma^{(1)}$, $\Sigma^{(7)}$, respectively, as well as the dynamics of their associated neighboring subsystems $\left\{\Sigma^{(2)}, \Sigma^{(3)}\right\},\left\{\Sigma^{(4)}, \Sigma^{(6)}\right\}$, while $\Sigma^{s}$ is less affected by these faults. The use of the proposed distributed adaptive FTC scheme, contributed to the successful compensation of the sensor faults effects on the local and neighboring dynamics of subsystems $\Sigma^{(1)}, \Sigma^{(7)}$.

\section{CONCLUSIONS AND FUTURE WORKS}

In this paper we proposed the design of a distributed adaptive fault tolerant control scheme for HVAC systems that may be affected by multiple sensor faults. Exploiting the HVAC system topology, a bank of local monitoring agents were designed to detect, isolate and estimate the isolated sensor faults. In order to compensate the sensor fault effects, the estimation of sensor faults is used by neighboring control agents in order to compensate the effects of the faults of the isolated sensors. A simulation example illustrates the effectiveness of the proposed scheme, which is applied to a 7-zone HVAC system. Future work will involve the rigorous analysis of stability and tracking performance of the proposed distributed reconfigurable control scheme.

\section{REFERENCES}

[1] M. Blanke, M. Kinnaert, J. Lunze, and M. Staroswiecki, Diagnosis and Fault-Tolerant Control. Springer Verlag Berlin Heidelberg, 2006.

[2] G. Escrivá-Escrivá, I. Segura-Heras, and M. Alcázar-Ortega, "Application of an energy management and control system to assess the potential of different control strategies in hvac systems," Energy and Buildings, vol. 42, no. 11, pp. 2258-2267, 2010.

[3] H. Doukas, K. D. Patlitzianas, K. Iatropoulos, and J. Psarras, "Intelligent building energy management system using rule sets," Building and Environment, vol. 42, no. 10, pp. 3562-3569, 2007.

[4] Z. Du and X. Jin, "Tolerant control for multiple faults of sensors in vav systems," Energy conversion and management, vol. 48, no. 3, pp. 764-777, 2007.

[5] S. Wang and Y. Chen, "Fault-tolerant control for outdoor ventilation air flow rate in buildings based on neural network," Building and Environment, vol. 37, no. 7, pp. 691-704, 2002.

[6] V. Reppa, P. Papadopoulos, M. M. Polycarpou, and C. G. Panayiotou, "A distributed virtual sensor scheme for smart buildings based on adaptive approximation," in 2014 International Joint Conference on Neural Networks (IJCNN), 2014, pp. 99-106.

[7] _ - "A distributed architecture for HVAC sensor fault detection and isolation," IEEE Transactions on Control Systems Technology, vol. 23, pp. 1323-1337, 2015.

[8] P. M. Papadopoulos, V. Reppa, M. M. Polycarpou, and C. G. Panayiotou, "Distributed Adaptive Estimation Scheme for Isolation of Sensor Faults in Multi-zone HVAC Systems," in Proceedings of 9th IFAC SAFEPROCESS, 2015, pp. 1146-1151.

[9] M. Zaheer-Uddin and R. Patel, "Optimal tracking control of multizone indoor environmental spaces," Journal of dynamic systems, measurement, and control, vol. 117, no. 3, pp. 292-303, 1995.

[10] E. Witrant, S. Mocanu, and O. Sename, "A hybrid model and MIMO control for intelligent buildings temperature regulation over WSN," in 8th IFAC Workshop on Time Delay Systems, 2009.

[11] V. Reppa, M. M. Polycarpou, and C. G. Panayiotou, "Adaptive approximation for multiple sensor fault detection and isolation of nonlinear uncertain systems," IEEE Transactions on Neural Networks and Learning Systems, vol. 25, no. 1, pp. 137-153, 2014.

[12] — - "Distributed sensor fault diagnosis for a network of interconnected cyberphysical systems," Control of Network Systems, IEEE Transactions on, vol. 2, no. 1, pp. 11-23, 2015.

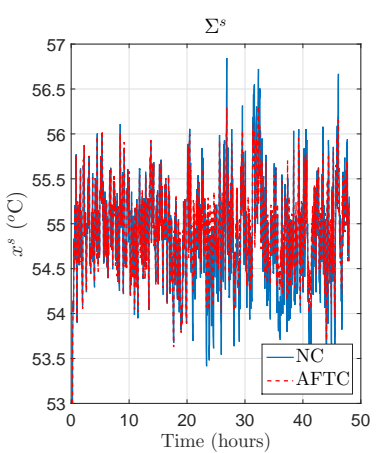

(a)

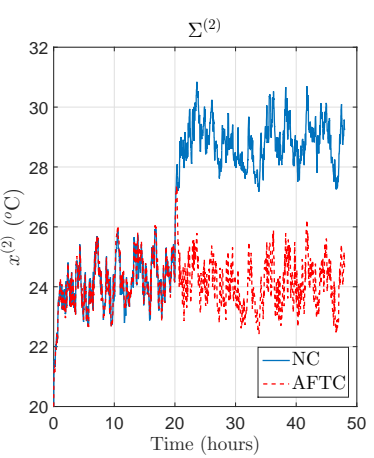

(c)

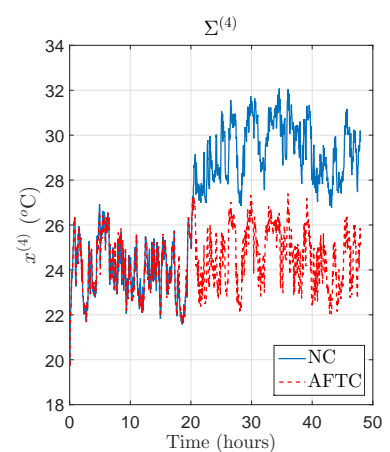

(e)

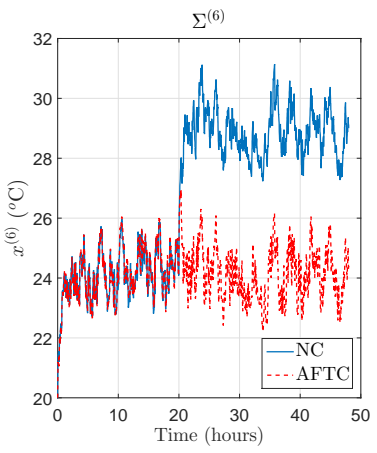

(g)

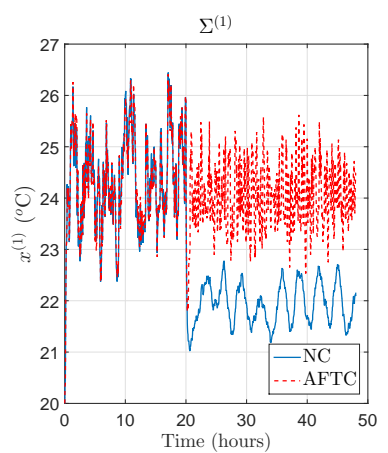

(b)

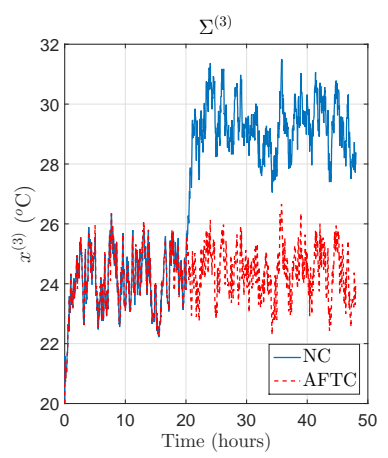

(d)

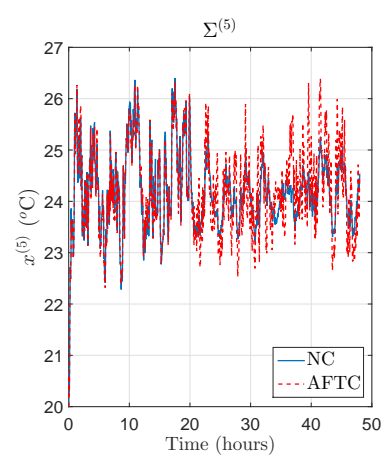

(f)

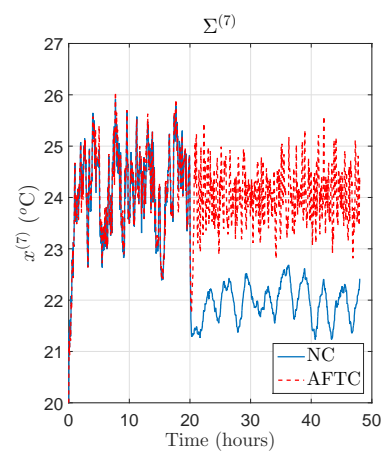

(h)
Fig. 3. HVAC system temperature response in multiple simultaneous bias sensor fault scenario in sensors $S^{(1)}, S^{(7)}$ : controlled by the nominal control (NC) scheme (blue solid line), controlled by the adaptive fault-tolerant control (AFTC) scheme (red dashed line) 